\title{
Die Zerstörung der Grabeskirche: Anfragen an orientchristliche Quellen
}

\section{DOROTHEA WEL'TECKE}

\section{Einleitung}

Jerusalem ist für die orientalischen Christen ein heiliger Ont höchster Bedeutung. Das gilt auch für die Grabeskirche. Damit ist noch nichts darüber gesagt, in welcher Weise die Stadt bedeutsam ist. Jerusalem kann einfach als Symbol in Liturgie, Kunst und Frömmigkeit erinnert und gefeiert werden. Sowohl christliche wie jüdische Jerusalemfrömmigkeit kommt deshalb zu Zeiten völlig ohne den Bezug auf den realen Ort aus. Aber die Stadt kann auch physisch aufgesucht und crinnert werden. Dann mag auch ihr zeitgenössisches Ergehen relevant sein. Und schließlich können das geographische Jerusalem und sein zeitgenössischer Zustand wiederum auf die symbolische Bedeutung und Darstellung zurückwirken. Für diese unterschiedlichen Ebenen der Jerusalem- und Grabeströmmigkeit in den orientalischen Kirchen ist die Grundlagentorschung verschieden weit gediehen. Doch gilt generell, dass sie sich nicht mit der intensiven Forschung über die lateinischen Christen messen kann. Dic Antworten müssten spezifisch ausfallen. Zudem wäre zeitlicher Wandel zu berücksichtigen.

Der Terminus „orientchristlich“ bezieht sich hicr auf Christen jener östlichen Kirchen, die am theologischen Prozess der Reichskirche und an den Konzilien spätestens nach dem Konzil von Chalkedon im Jahr 451 nicht mehr beteiligt waren. Sie bezeichnen sich heute oft als „altorientalische Kirchen“, um sich unter anderem von den arabisch sprechenden Christen griechisch-orthodoxer oder katholischer Konfession zu unterscheiden. Dazu gehören konfessionell zum einen die Kirchen der miaphysitischen Tradition. Sie umfasst die syrischorthodoxe (veraltet: jakobitische), die armenisch-apostolische Kirche, die koptische Kirche und die äthiopische Kirche. Zum anderen versteht man darunter die Orthodoxe Kirche des Ostens (veraltet: nestorianische), als östlichen Zweig der syrischen Tradition. Diese Kirche formierte sich auf sassanidischem Reichsgebiet.

Das Verhältnis der orientalischen Christen zu Jerusalem wurde in verschicdenen Studien erforscht.' Die Arbeiten haben sich darauf konzentriert, die mate-

1 U. a. E. Cerulli, Etiopi in Palestina, Storia della comunità etiopica di Gerusalemme I, Rom 1943; O. Meinardus, The Copts in Jerusalem, Kairo 1960; J.-M. Ficy, Pour un Oriens Christianus Novus. Répertoire des diocèses Syriaq̨ues orientaux et occiden- 
riellen Grundlagen der physischen Präsenz der orientalischen Christen in Jerusalem und die Bedeutung des geographischen Ortes zu klären. Auf diese Ergebnisse kann hier zurückgegriffen werden. Sie sollen deshalb zunächst kurz skizziert werden. In cinem zweiten Schritt werden orientchristliche Quellen auf den Niederschlag untersucht, den die Zerstörung der Grabeskirche dort hinterlassen hat. Dabei sind zwei dieser Quellen - die Geschichte der koptischen Patriarchen des sogenannten Sawīus Ibn-al-Mulkaffac und die Chronik des syrisch-orthodoxen Gelelarten und Kirchenfürsten Bar ${ }^{\complement}$ Ebrōyō - in einem zentralen Aufsatz von Marius Canard bereits aufgegriffen worden.? Damit ergibt sich drittens die Aufgabe, diese beiden Problemfelder miteinander zu verknüpten. Erstens ist zu prüfen, ob sich aus diesen christlichen Quellen neue Aspekte in Bezug auf die T'hesen Canards ergeben. Zweitens ist zu fragen, wie sich im Prisma der Zerstörung der Grabeskirche das Verhältnis der orientalischen Kirchen zu Jerusalem und das Verhältnis zwischen den religiösen Gemeinschaften in dieser Zeit darstellen.

\section{Die Bedeutung Jerusalems für die altorientalischen Christen}

Die symbolische Bedeutung Jerusalems in Liturgie und Spiritualität steht außer Frage. Dabei wäre hier zwischen den Kirchen stärker zu differenzieren, als es in diesem Rahmen möglich ist. Allen orientalischen Kirchen ist jedoch gemeinsam, dass ihre Jerusalemmystik sich nicht aus denselben Quellen speist, die für dic lateinische Kirche prägend geworden sind. Die für letztere so zentrale Apokalypse Johannis und ihre Beschreibung des Himmlischen Jerusalem hat in den östlichen Kirchen nicht denselben Rang. Die syrisch-orthodoxe Kirche hat das Buch nicht einmal in den Kanon aufgenommen. Eine weitere Gemeinsamkeit ist die Tatsache, dass Jerusalem kirchenpolitisch keine zentrale Stellung einnahm und somit institutionell hinter anderen Städten mit Sitzen von Patriarchaten beziehungsweise Katholikaten rangierte. Die orientalischen Kirchen hatten die

taux, Stuttgart 1993 (Beiruter Texte und Studien 49); A. Palmer, 'T'he Fistory of the Syrian Orthodox in Jerusalem, in: OCP 75 (1991) 16-43; J. Pahlitzsch, St. Maria Magdalena, St. Thomas und St. Markus. Tradition und Geschichte dreier syrisch-orthododar Kirchen in Jerusalem, in: OCP 81 (1997) 82-106; H-I. Kaufhold, Zur Bedeutung Jerusalems für die syrisch-orthodoxe Kirche, in: W. Brandmüller ( Hg. $_{\text {. }}$, L'idea di Gerusaletnme nella spiritualità cristiana del medioevo, Città del Vaticano 2003 (Pontificio comitato di scienze storiche. Atti e documenti 12), 132-165; O. Limor-G.G. Stroumsa ( $\mathrm{Hg}$.), Christians and Christianity in the Holy Land. From the Origins to the Latin Kingdoms, Turnhout 2006 (Cultural Encounters in Late Antiquity and the Middle Ages 5 ).

2 M. Canard, La Destruction de l'Église de la Résurrection par le calife Hāankim et l'histoire de la descente du feu sacré, Byz 35 (1965) 16-43, besonders 17 und 35-38.
Erhebung Jerusalems zum Patriarchat auf dem Konzil von Chalkedon im Jahr 451 nicht anerkannt, sondern die Diözesanstruktur der vorchalkedonensischen Zeit mehr oder weniger konserviert. Das syrisch-orthodoxe Bistum von Jerusa. lem beispielsweise konnte zwar gelegentlich chrenhalber auch einmal Patriarchat genannt werden, wie Hubert Kaufhold gezeigt hat. ${ }^{3}$ Aber die Bischöfe beziehungsweise Metropoliten von Jerusalem konnten nicht den 'Titel Patriarch füh ren. Und erst seit 1311 trägt der armenische Bischof den Titel Patriarch, ohne damit denselben Rang einnehmen zu können wie das Oberhaupt der armenischen Kirche, der Katholikos."

Wie in altchristlicher Zeit sahen um das Jahr 1000 nicht alle Theologen das körperliche Aufsuchen des Ortes als heilsam oder auch als nötig an. Dennoch war Jerusalem für Pilger aller Konfessionen ein wichtiges Pilgerziel, ebenso wie die anderen Loca Sancta im Heiligen Land. Selbst aus dem fernen Beijing machten sich im späten 13. Jahrhundert noch zwei Mönche der Kirche des Ostens mit dem erklärten Ziel auf, Jerusalem und die heiligen Stätten dort zu sehen. ${ }^{5}$ Allerdings wurde die Grabeskirche von der Reichskirche, aus der Perspektive der Orientalen also von Häretikern, dominiert. Pilger anderer Konfessionen mussten sich an der Grabeskirche dem Rhythmus und der Ordnung des reichskirchlichen Klerus unterwerfen, auch wenn sie später eigene Kapellen unterhalten durften. So weit orientalische Kirchen von der Liturgie an der Grabeskirche beeinflusst worden waren, ging dieser Einfluss noch auf die Zeit vor der Konfessionalisierung im 5. und 6. Jahrhundert zurück; die reichskirchliche Gegenwart wirkte auf sie nicht mehr ein

Die orientalischen Kirchen besaßen darüber hinaus in Jerusalem noch ihre eigenen Klöster, in denen sie die Pilger beherbergen konnten und in denen die Bischöfe residierten, sofern Jerusalem ein Bischofssitz war. Dabei unterstanden die syrisch-orthodoxen, die koptischen und die äthiopischen Gläubigen dem syrisch-orthodoxen Bischof, weil sich diese Kirchen als Einheit verstanden und grundsätzlich auf Doppelbesetzungen verzichteten. Entsprechend waren die syrisch-orthodoxen Christen in Ägypten der koptischen Hierarchie zugeordnet.

3 Kaufhold, Jcrusalem (s. Anm, 1).

$4 \mathrm{Zu}$ den Armeniern in Jerusalem vgl. M. E. Stone (Hg.), The Armenians in Jerusalem and the Holy Land, Leuven 2002; C. Mutafian, Les Arméniens et Jérusalem au Moyen Âge, in: Revue Arménienne des Questions Contemporaines 4 (2006) 9-18; S. La Porta, The Armenian Episcopacy in Mamluk Jerusalem in the Aftermath of the Council of Sis (1307), in: Journal of the Royal Asiatic Society of Great Britain and Ireland 17 (2007) 99-114.

5 A. Toepel (Hg.), Die Mönche des Kublai Khan. Die Reise der Pilger Mar Yahballaha und Rabban Sauma nach Europa, Darmstadt 2008; P. Bedjan (Hg.), Histoire de Mar Jabalaha, de trois autres patriarches, d'un prêtre et de deux läques nestoriens, Paris Leipzig 1895. 
Die armenische Kirche unterhielt ein eigenes Bistum. Gläubige der Kirche des Ostens hatten um das Jahr 1000 wahrscheinlich keinen eigenen Bischof in Jerusalem."

Eine nennenswerte Anzahl ortsansässiger orientalischer Christen gab es, im Unterschied zur Reichskirche, allem Anschein nach nicht. Größere Kolonien unterhielt nur die armenische Kirche um die Kirche des Hl. Jakob. Die übrigen waren nur mit wenigen Mönchen und Klerikern vertreten. Das syrisch-orthodoxe Kloster Maria Magdalena zum Beispiel, das zur Zeit der Zerstörung der Grabeskirche und mindestens bis ins 13. Jahrhundert bestand, konnte sich wegen seiner geringen Zahl an Bewohnern und seiner dürftigen Ausstattung auch in seiner kulturellen Bedeutung nicht mit den berühmten syrisch-orthodoxen Klöstern am Euphrat messen.7 Insgesamt sind die Quellen für die Anwesenheit orientalischer Christen in Jerusalem äußerst karg, so dass vielfach keine genaue Vorstellung möglich ist.

Für die orientalischen Kirchen war Jerusalem also um das Jahr 1000 weder ein kirchenorganisatorisches, noch ein kulturelles oder theologisches Zentrum. Die Jerusalemfrömmigkeit und die Liturgie kamen ohne die konkrete Stadt aus. Andere Städte wurden als konkrete Erinnerungsorte vielleicht sogar mehr verehrt als Jerusalem. Genannt werden sollte an dieser Stelle etwa die Stadt Edessa, die als gesegnet galt und auch für die kulturelle Identität der syrisch-orthodoxen Christen zentral war.

\section{Ein Blick in die Quellen}

Marius Canard arbeitete im Zusammenhang mit der Zerstörung der Grabeskirche einen wichtigen Aspekt heraus, der die Beziehungen zwischen den Religionen in dieser 'Zeit und an diesem Ort besonders charakterisiert. Die Oster-

6 M. LeQuien, Oriens Christianus, in quatuor patriarchatus digestus; quo exhibentur ecclesiae, patriarchae, cacterique praesules totius Orientis, I-III, Paris 1740; Neudruck: Graz, 1958; Fiey, Oriens (s. Anm. 1).

7 Kaufhold, Jerusalem (s. Anm. 1) 157.

8 Zu Edessa vgl. P. Devos, Égérie à Ĺdesse. S. 'Thomas l'Apôtre, Le Roi Abgar, in: AB 85 (1967) 380-400; H. J. W. Drijvers, Addai und Mani. Christentum und Manichäismus im dritten Jahrhundert in Syrien, in: R. Lavenant, S. J. (Hg.), III. Symposium Syriacum 1980. Les contacts du monde syriaque avec les autres cultures (Goslar 7-11 Septembre 1980), Rom 1983 (OCA 221), 171-185; J. B. Segal, Edessa. 'The Blessed City, Oxford 1970; A. Desreumaux, La Doctrine d'Addai. Essai de classement des témoins syriaques et grecs, in: Augustinianum 23 (1983) 181-186; D. Weltecke, Die „Beschreibung der Zeiten" von Mor Michael dem Großen (1126-1199). Eine Studie zu ihrem historischen und historiographiegeschicbtlichen Kontext, Louvain 2003 (CSCO 594, Subsidia 110), 213, 224f., 237-238. liturgie an der Grabeskirche war um die Jahrtausendwende ein interreligiöses Fest! Er stellte vor allem muslimische Quellen zusammen, die berichten, in welcher Weise die muslimische Bevölkerung, der Emir von Jerusalem, der Imam der Moschee und, über Boten, sogar der Kalif im fernen Bagdad auf das wunderbare Erscheinen des Heiligen Feuers in der Osternacht warteten. Die muslimischen Obrigkeiten übten dabei einige kontrollierende und leitende Funktionen aus, indem sie zum Beispiel die T'ür zum Grab schlossen oder sich in dessen nächster Nähe postierten. Dabei wurde die Zeit der Feuererscheinung von den Muslimen offenbar als Omen benutzt, von dem man sich Aufschluss über den Verlauf des kommenden Jahres erhoffte."

Canard sammelte muslimische Quellen, die betrügerische Machenschaften der Christen in der Osternacht vermuteten. Sie entwickelten unterschiedliche Theorien, wie das Feuer ins Werk gesetzt wurde. Einige Quellen bringen diese Betrugsvorwürfe mit dem Kalifen al-Häkim in Zusammenhang. Hier wird der Kalif von einer Gaukelei der Christen im fernen Jerusalem in Kenntnis gesetzt. Daraufhin beschließt er die Zerstörung der Kirche. Canard schlug daher vor, dass in der Tat gerade die muslimische Teilnahme am Osterfest eine Ursache für die Zerstörung der Kirche gewesen sein könnte. ${ }^{10}$ Schließlich versuchten muslimische Geistliche oder Obrigkeiten atuch sonst - oft vergeblich - die Teilnahme von Muslimen an christlichen Festen oder das Wallfahren zu christlichen Heiligen Orten zu unterbinden."

Seine Beobachtungen wurden von Benjamin Kedar vor einigen Jahren aufgegriffen und in den größeren Kontext interreligiöser Begegnungen an Heiligen Orten eingebettet. ${ }^{12}$ Er forderte, diese interreligiösen Begegnungen soziologisch zu differenzieren, je nach dem, wer das Geschehen an einem Ort bestimmte und ob die Beteiligten gleichberechtigt agieren konnten. Bei der Untersuchung der Feierlichkeiten in der Grabeskirche folgt aus seinen Vorschlägen, dass nicht nur zwischen Christen einerseits und Muslimen andererseits unterschieden werden darf, sondern auch die Konfession als eine eigene Kategorie berücksichtigt werden muss. Denn um 1000 befanden sich Konfessionen in der Grabeskirche, die nur Zuschauer waren. Anders als der Emir oder der Imam waren sie von der eigenständigen Mitwirkung, ganz zu schweigen von einer Kontrolle der Feierlichkeiten, ausgeschlossen.

9 Canard, Destruction (s. Anm. 2) 20-24; 34-38

10 Canard, Destruction (s. Anm. 2) 39-42.

11 Z.B. B. Shoshan, Popular Culture in Medieval Cairo, Cambridge 1993 (Cambridge Studies in Islamic Civilization), 68 u.

12 B. Z. Kedar, Convergence of Oriental Christian, Muslim, and Frankish Worshippers The Case of Saydnaya, in: Y. Hen ( Hg.), De Sion exibit lex et verbum domini de Hierusalem: Essays on medieval law, liturgy, and literature in honour of Amnon Linder, Brepols 2001 (Cultural Encounters in Late Antiquity and the Middle Ages), 59-69. 


\section{Michael von Tinnis}

Die älteste orientchristliche Quelle, die über die Zerstörung der Grabeskirche berichtet, stammt aus der Geschichte der koptischen Patriarchen des sogenannten Sawïrus Ibn-al-Mukaffa'.13 Dieser Bericht zur Zerstörung der Grabeskirche soll nun zuerst in seinem Kontext betrachtet werden. Die Patriarchengeschichte ist eine in mehreren Fortsetzungen und Überarbeitungen entstandene Folge von Patriarchenbiographien, an der unterschiedliche Autoren gearbeitet haben. ${ }^{14} \mathrm{Der}$ hier relevante fünfte Bericht stammt von einem Bischof Michael von 'Tinnis, einem Zeitgenossen der ersten Hälfte des 11. Jahrhunderts. Er behandelt die Regierungszeit des koptischen Papstes Zacharias (1004-1032). In seinen ausführlichen Bericht schaltet sich der Bischof immer wieder ein, zumeist als Ohren zeuge von mündlichen Berichten aus der Zeit des Kalifen al-Hākim. Einer der Augenzeugen, mit denen er sprach, war sein leiblicher Vater.

Als historischer Berichterstatter ist er wohl seinem bekannten melkitischen Zeitgenossen Yahya von Antiochia (gest. ca. 1066) unterlegen, zu dem später zuruickzukehren sein wird. ${ }^{15}$ Denn Bischof Michacls Darstellung ist weniger ein Bericht des Geschehens als dessen spirituelle Verarbeitung. Hier soll indessen nicht so sehr der historische Gehalt seiner Darstellung geprüft werden als vielmehr Michaels Interpretation des Geschehens im Vordergrund stehen. Die Ereignisse sind bei ihm von hagiographischen Elementen und Wundern umankt, in denen sich fromme Kopten unter dem Druck der Verfolgungen bewähren und Christus umgekehrt den Gequälten beisteht. ${ }^{16}$ Die diskriminierenden Maßnahmen gegen Juden und Christen bewirken in der Darstellung des Bischofs vor allem, dass sie als solche auf der Straße, dem Markt und im Bad erkannt werden

3 A. Suryal Atiya - Y. Abd al-Masih - O. H. E. Burmester, History of the patriarchs of the Egyptian Church, known as the history of the Holy Church by Sawirus Ibn-alMukaffa', Il, 2, translated and annotated, Kairo 1948 (Publications de la Société d'ArMukáffa', II, 2, trans
chéologie Copte)

14 G. Graf, Geschichte der christlichen arabischen Literatur, II. Die Schriftsteller bis zur Mitte des 15. Jahrhunderts, Vatikanstadt 1947 (Studie Testi 133), 300-318, 484; J. Den Feijer, Fistory of the Pacriarchs of Alexandria, in: A. Suryal Atiya, The Coptic Encyclopedia, 4 (1991), S. 1238-1242.

15 J. Kratchkovsky - A. Vasiliev (Bearb.), Histoire de Yahya-ibn-Sa Id d'Antioche. Continuateur de Sa 'id--ibn-Bitriq, éditée et traduite en français, II, in: R. Graffin - F. Nau (Hg.), Paris 1932 ( $P O 23)$, S. 141-312 (= 239-502).

16 Vgl. die Evaluierung im Vergleich zur jüdischen Erinnerung: M. Cohen, Persecution, Response, and Collective Memory: "The Jews of Islam in the Classical Period, in: D. Frank (Hg.), T'he Jews of Medieval Islam. Community, Society and Identity. Proceedings of a conference held by the Institute of Jewish Studies, University College London, Leiden 1995 (Études sur le judaïsme médiéval 16), 145-164, hier: S. 154, Nr. 29. sollen. ${ }^{7}$ Dass viele Menschen zumindest kurze Zeit auch zum Schein das Christentum verließen, wird ebenfalls berichtet, ebenso von der verbreiteten Versuchung, dies zu tun. Denn namentlich die wirtschaftlichen Nachteile der sozialen Abgrenzung der Christen seien sehr schnell spürbar geworden. Auch seien die Christen nun von Muslimen auf der Straße angefeindet und angespuckt worden; Michael nennt vor allem die Region um 'Tinnis, also seine Heimat und seinen Vater als Augenzeugen dafür, 18

Michael berichtet, dass während dieser diskriminierenden Maßnahmen eine Zahl Christen zum Beispicl durch grausame Prügel- und Amputationsstrafen auch das Martyrium erlitt. Diese sind durchweg hochgestellt und wählen unter dem Zwang, den Islam anzunehmen oder zu sterben, das zweite Schicksal.19 Nichtmuslime sollten nun keine Ämter mehr ausüben dürfen, so wie es ursprünglich die Bestimmungen für die religiösen Minderheiten vorgesehen hatten. Die Praxis im fatimidischen Ägypten hatte anders ausgesehen. ${ }^{20}$ Auch diese Martyrien erscheinen in Michaels Bericht demnach nicht als Folge wahloloser antichristlicher Maßnahmen. Stattdessen ergeben sie sich aus dem Versuch, den administrativen Apparat zu muslimisieren beziehungsweise die religiöse Vermischung in demselben zu beenden. Diese Politik sollte unter den Ayyubiden und Marnluken aufgegriffen werden und schließlich die Christen aus der Verwaltung verdrängen.

Der Bischof betont, dass der Kalif al-Hākim, erschreckend groß- und blauäugig, gleich einem blutgierigen Löwen, ${ }^{21}$ wenig am Prunk interessiert und in einfacher Kleidung auf einem Esel reitend, zugleich auch alle muslimischen Bewohner Ägyptens in Schrecken versetzt habe. Seine Aktionen hätten sich zuerst gegen arabische Adelige und Militärs gerichtet, dann gegen die Schreiber in der Verwaltung, schließlich auch gegen gewöhnliche Muslime. Vor allem die Frauen als Adressaten von Anordnungen werden besonders hervorgehoben. Frauen hätten das Haus nicht mehr verlassen, zum Bad gehen oder auf dem Markt tätig sein dürfen, um ihren Lebensunterhalt zu verdienen, ${ }^{22}$

17 Allgemein zu den Edikten al-Hăkims H. Halm, Der Treuhänder Gottes. Die Edikte des Kalifen al-Häkim, in: Der Islam 63 (1986) 11-72.

18 Atiya - Abd al-Masīh - Burmester, History (s. Anm. 13), cngl. Üs. S. 201-204.

19 Atiya - Abd al-Masĭ - Burmester, History (s. Anm. 13) 184-186.

20 Neuerdings dazu M. Brett, Population and Conversion to Islam in Egypt in the Medieval Period, in: U. Vermetlen - J. van Steenbergen (Hg.), Egypt and Syria in the Fatimid, Ayyubid and Mamluk Eras, IV, Leiden 2005, 1-32, hier 23 und andere Beiträge dieses Bandes ebenso wic dic übrigen Bände dieser Veranstaltungsteihe.

21 Atiya - Abd al-Masī - Burmester, Kistory (s. Anm. 13) 1866

22 Atiya --. Abd al-Masith -- Burmester, History (s. Anm. 13) 188, 192; vgl. zu Frauen unter den Fatimiden D. Cortese - S. Calderini, Women and the Fatimids in the World of Islam, Edinburgh 2006, hier besonders 192-208. 
Die Eingriffe des Kalifen in das Leben der Christen sind bei Michael von 'T'innis in ein pecatis exigentibus Motiv eingebunden. Der Bischof glaubt, dass Gott die Kopten für ihr unfrommes Verhalten strafte. Er benennt den Änterkauf als konkrete Verfehlung. Nicht mehr die fähigsten Mönche hätten Bistümer erhalten, sondern allenfalls diejenigen, die kräftig dafür zahlten. ${ }^{23}$ Hinzu kam die unheilvolle Macht der Bischöle über den heiligen, aber entscheidungsschwachen Papst Zacharias, ${ }^{24}$ der seine Berater ungehindert habe gewähren lassen. Diese Konstellation habe die Wut eines ehrgeizigen Mönches Johannes aus dem Macariuskloster heraufbeschworen, der cin Bistum für sich forderte. ${ }^{25}$ Dieser fühlte sich als armer Mann zurückgesetzt und wandte sich deshalb mit Briefen an den Kalifen al-Hākim, um sich über den Patriarchen und die unrechtmäßigen Praktiken zu beschweren. Während christliche Bedienstete die Weiterleitung zunächst verhindert hätten und die Familie des Patriarchen den Mann habe steinigen lassen, seien die Briefe schließlich doch vor den Kalifen gelangt, ${ }^{26}$ der sich bereits als unberechenbar und unduldsam erwiesen habe.

Die Beschwerdebriefe hätten nun die Verfolgungen von Christen ausgelöst, die mit der Gefangensetzung des Papstes Zacharias einen Höhepunkt erreichten. Zu späterer Zeit, als die Verfolgungen bereits im Abebben begriffen waren, habe dieser Mönch, der die 'Tortur überlebt hatte, erneut lautstark ein Bistum für sich gefordert. Wieder wird bekräftigt, dass er allein die Verfolgungswelle ausgelöst habe, da er den Zorn des aufbrausenden Kalifen auf die Christen gezogen hatte. ${ }^{27}$ Dass diese Erklärung nicht ganz logisch ist und gewisse chronologische Probleme mit sich bringt, scheint den Erzähler nicht zu stören. Er will die macht-- und religionspolitischen Aktionen des Kalifen gegen die verschiedenen Religionsgemeinschaften und gegen die Frauen also nicht auf eine einzige Ursache zurückführen wie etwa auf die durchaus beschriebene religiöse Radikalität und die emotionale Erregbarkeit des Kalifen. Zwar charakterisiert Michael den Kalifen ferner durch ein Interesse für Astrologie und andere, als irreführend gekennzeichnete Wissenschaften. ${ }^{28}$ Aber er erklärt nur die allgemeine Politik mit dem Charakter des Kalifen, die Maßnahmen gegen die Kopten jedoch mit deren eigenem Handeln.

Nachdem er den Patriarchen wegen des ungerechten Handelns gegenüber dem Mönch verhaftet hätte, habe der Kalif die vollständige Zerstörung der Grabeskirche befohlen. Sie sollte dem Erdboden gleich gemacht werden. ${ }^{29}$ Michael

23 Atiya - Abd al-Masth - Burmester, History (s. Anm. 13) 180.

24 Atiya - Abd al-Masil - Burmester, History (s. Anm. 13) 180

25 Zu den Klöstern vgl. HE. Gerard - E. White, The Monasteries of the Wádi'n Natrun, I-III, New York 1926-1932.

26 Atiya - Abd al-Masih - Burmester, History (s. Anm. 13) 182, 193

27 Atiya - Abd al-Masin - Burmester, History (s. Anm. 13) 207.

28 Atiya - Abd al-Masih - Burmester, Fistory (s. Anm. 13) 187.

29 Atiya - Abd al-Masil - Burmester, History (s. Anm. 13) 194. erzählt, ein Sekretär, der der Kirche des Ostens angehörte und Ibn Šrmn hieß, habe die Ausfertigung des Befehls übertragen bekommen. Aus Gram über seine Feigheit, nicht das Martyrium auf sich zu nehmen und die Ausfertigung des Befehls zu verweigern, habe der Mann anschließend seine schuldige rechte Hand so lange gegen eine Wand geschlagen, bis sie zertrümmerte. Wenig später sei er gestorben. ${ }^{30}$ Es folgte die Zerstörung der restlichen Kirchen und ihre Plünde-rung. Hohe Steuern wurden erhoben. Zugleich wurde das Verbot erlassen, dem Handel nachzugehen. Mindestens ebenso schwer wie die Zerstörung der Grabeskirche, oder vielleicht noch schwerer, wiegt in diesem Bericht, dass die Christen die Eucharistie zunächst jahrelang nur noch in den Klöstern in der Wüste erhalten konnten, später heimlich in ihren Häusern. Die Menschen zogen von je her zu den Hochfesten in die Wüstenklöster, um mit den Mönchen zu feiern. In Gefahrenzeiten waren die Klöster der Rückzugsort. Auch der Papst Zacharias habe sich nach seiner mehrmonatigen Gefangenschaft, während derer er durch Schläge und die Drohung, den Löwen vorgeworfen zu werden, habe zum Übertritt zum Islam bewegt werden sollen, dorthin begeben. ${ }^{31}$

Der Bericht ist mit überraschenden Elementen durchsetzt, die wiederholt aus verschiedenen Perspektiven mitten in der Verfolgungszeit funktionierende Beziehungen zwischen Christen und Muslimen und auch zwischen dem Kalifen und den Christen benennen. So versuchte ein (namentlich genannter) Diakon in dieser Zeit cin freiwilliges Martyrium zu provozieren, indem er Christus öffentlich lobte. Er behielt jedoch nicht nur sein Leben, sondern gewann sogar vermittelnden Einfluss auf den Kalifen. Vielen Menschen habe er in Schuldgefangenschaft und anderen Kalamitäten helfen können. ${ }^{32}$ Immer wieder werden einzelne Muslime mit Namen genannt, die mit Christen befreundet waren und diese aus Gefangenschaft und Todesangst erretteten oder sich beim Kalifen erfolgreich für sie einsetzten. ${ }^{33}$ Als gegen Ende der Verfolgungszeit apostasierte Christen und

30 Atiya - Abd al-Masị - Burmestex, History (s. Anm, 13) 194. Ein hoher nestorianischer Beamter, genannt 'Tsa b. Nesțürus ist auch andernorts bezeugt, vgl. N. A. Stillman, 'The Jews of Arab lands. A history and source book, Philadelphia 1979, 200; Kratchkovsky - Vasiliev, Histoire de Yahya (s. Anm. 15) S. 239, 241 u. ö, s. u. P. Bedjan (Hg.) Gregorii Barhebraei Chronicon Syriacum, Paris 1890 (syr.); E. A. Wallis Budge (Üs./Hg.), The Chronography of Gregory Abû'l Faraj, the son of Aaron, the hebrew physician commonly known as Bar Hebraeus being the first part of his political history of the world, I-II, Oxford/London 1932 (engl.), S. 200 (180). Doch wurde dieser Mann zu Beginn der Amtszeit des Kalifen bereits umgebracht. Auch wegen des Namens muss hier jemand anders gemeint sein. Seiner Identität kann hier nicht nachgegangen werden.

31 Atiya - Abd al-Masīh - Burmester, History (s. Anm. 13) 201-207.

32 Atiya - Abd al-Masī - Burmester, History (s. Anm. 13) 195-197, 201.

33 Atiya - Abd al-Masih- - Burmester, History (s. Anm. 13) $199 \mathrm{f}$. 
Juden vom Kalifen die Erlaubnis zur Rückkehr in thre angestammte Religion erbaten, wurde ihnen dies entgegen dem Apostasieverbot zugestanden. Sie hätten sich nur bereitfinden müssen, die diskriminierenden Erkennungszeichen - hölzerne Kreuze für Christen und Kugeln für Juden - wieder anzulegen. ${ }^{34}$ Ein Mönch habe sich diesen Apostaten angeschlossen. Er erwirkte vom Kalifen die Erlaubnis, ein Kloster zu bauen. Noch dazu habe dieser oft das Kloster besucht, um mit den Mönchen ihr asketisches Mahl zu teilen. Wer dem Kalifen ein Anliegen vorzutragen hatte, habe sich an diesen Mönch gewandt. ${ }^{35}$ Diese Elemente zeigen an, dass der Bischof keine einfache, antimuslimische Polemik beabsichtigte. Vielmehr wollte er immer wieder zeigen, dass die christliche Standhattigkeit und die Bereitschaft zum Martyrium am Ende nicht zuletzt von den Muslimen, ja vom Kalif selbst bewundert wurden. ${ }^{36}$ Wenn die Schuld der Christen das Verhängnis heraufbeschworen hatte, war es ihre Trete zum Christentum, die schließlich auch belohnt wurde.

Deshalb stehen am Schluss dieser Verfolgungszeit nicht zufällig eine vollständige Versöhnung zwischen dem Kalifen al-Hälkim, den Christen und dem Patriarchen, die Aufhebung aller Maßnahmen und die Restauration aller Kirchen. ${ }^{37}$ Am Ende suchte der Kalif das Macariuskloster auf und diskutierte mit den Bischöfen über ihre Treue zum Patriarchen, um schließlich die Tugendhaftigkeit und Standhaftigkeit des christlichen Glaubens anzuerkennen. Schließlich verschwand er eines Nachts und ward nie wieder gesehen. Von den bekannten Gerüchten um Mordkomplotte gegen den Kalifen berichtet Michael nichts. Damit zeichnet Michaels Bericht übrigens ein ähnlich widersprüchliches Bild des Kalifen - zwischen gerechtem Asket und unberechenbarem Verfolger - wie es die jüdischen Quellen ergeben. ${ }^{38}$

Das Motiv, das bei Michael von Tinnis die Verfolgungen letztlich auslöste, ist ein öfter begegnendes Element in orientchristlichen Chroniken: Kirchenmitglicder oder Kleriker, die als moralisch verdächtig dargestellt werden sollen, wenden sich in einer Streitsache um ihres Vorteiles oder ihres Ehrgeizes willen an die muslimische Obrigkeit und lösen dadurch Gefahren und ungerechte Maßnahmen aus. ${ }^{39}$ Damit ließen sich Christenverfolgungen durch die eigene Schuld

34 Atiya - Abd al-Masì - Burmester, History (s. Anm. 13) 205, so auch unten, Bedjan, Barhebraei Chronicon / Budge, Chronography Bar Hebracus (s. Anm. 30) S. 204 (184).

35 Atiya - Abd al-Masïh-Burmester, History (s. Anm. 13) 205.

36 Atiya - Abd al-Masih - Burmester, History (s. Anm. 13) 205-207.

37 Atiya - Abd al-Masih - Burmester, History (s. Anm. 13) 208.

38 Vgl. Stillman, Jews (s. Anm. 30) 200f.; Cohen, Persecution (s. Anm. 16) 154.

39 Vgl. J. B. Abbeloos - T. J. Lamy (Hg.), Gregorii Barhebrei Chronicon Ecclesiasticum, I-III, Louvain 1872, Paris 1874, 1877, III, S. 347-351 (syr.), 348-352 (lat.). erklären. Allerdings reflektiert dieses Motiv auch realistisch ein Element christlichen (und jüdischen) Lebens unter muslimischer Herrschaft. Es gehörte zu den üblichen Strategien in einem Konflikt, sich in der eigenen Sache auch an die außerkirchliche Obrigkeit zu wenden. ${ }^{* 0}$ Und tatsächlich scheint es, dass dabei gelegentlich die Stabilität der muslimischen Herrschaft oder der eigenen Position falsch eingeschätzt wurde, sei es in Ägypten, sei es in Bagdad oder'Takrit. Solche Aktionen konnten deshalb ungeplante zerstörerische Folgen haben und in Ausschreitungen gegen Christen enden. Festzuhalten ist hier ferner, dass Michael von Tinnis auch die Zerstörung der Grabeskirche nur mit innerägyptischen Vorgängen bzw. mit dem unkanonischen Verhalten des koptischen Klerus verknüpft; dic von Canard angesprochenen interreligiösen Interaktionen an der Grabeskirche erwähnt er nicht.

\section{Elias von Nisibis}

Ein weiterer Zeitgenosse der Ereignisse war ein Bischof der Kirche des Ostens, Elias von Nisibis (975-1046). ${ }^{41}$ Er verfasste unter anderem eine Weltchronik, die die wichtigsten Ereignisse und die wichtigsten Zeitrechnungen übersichtlich zusammenfassen sollte. Er berichtet, dass das Jahr $400 \mathrm{H}$. am 25. Ab des Jahres 1320 seleukidischer Zeitrechnung (d. i. das Jahr 1009 n. Chr.) begann. An diesem Tag habe der König von Ägypten befohlen, die "große Kirche in Jerusalem" zu zerstören und die Christen zu verfolgen. Überdies habe er daraufhin in seinem Königreich begonnen, sowohl christliche Kirchen wie jüdische Synagogen niederzureißen. ${ }^{42}$ Das Datum ist sehr detailliert; Elias scheint einen Zusammenhang zwischen der runden Jahreszahl und dem Beginn der Verfolgungen nahezulegen. An anderer Stelle berichtet Elias, dáss der Metropolit von Damaskus in diesen Jahren wegen der Verfolgungen gegen die Christen im Westen von seinem Sitz floh und nach Osten eilte, wo er dann auch eingesetzt worden sei. ${ }^{43}$ Motive, Wer-

40 Appelation von Muslimen durch Christen und Juden untersucht systematisch U. Simonsohn, Communal Botndaries Reconsidered: Jews and Christians Appealing to Muslim Authorities in the Medieval Near East, in: Jeqvish Studies Quarterly 14/4 (2007) 328-363 und weitere; zu innerchristlichen Konflikten, in die welt liche Obrigkeiten hereingezogen werden vgl. auch J. Pahlitzsch - D. Weltecke, Konflikte zwischen den nicht-lateinischen Kirchen im Königreich Jerusalem, in: D. Bauer - K. Herbers - N. Jaspert (Hg.), Jerusalem itn Hoch- und Spätmittehalter. Konflikte und Konfliktbewältigung - Vorstellungen und Vergegenwärtigungen, Frankfurt - New York 2001, 119-145.

41 E. W. Brooks / J.-B. Chabot ( $\mathrm{Hg} . / \mathrm{U} \mathrm{s}$.), Eliae metropolitae Nisibeni opus chronologicum, I-II, Paris 1909-1910 (CSCO 62-63, Scriptores Syri, 21m24).

42 Brooks - Chabot, Eline (s. Anm. 41) S. 225 (110)

43 Brooks - Chabot, Eliae (s. Anm. 41) S. 69-70 (35). 
tungen oder 'Tendenzen werden in diesem Bericht nicht deutlich; aber das ist auch sonst nicht die Aufgabe, die sich diese Chronik gestellt hat. Sie beschäftigt sich in der Hauptsache mit Synchronisierung und mit Zeitrechnung. "W

\section{Matthäus von Edessa}

Ganz anders ist die Darstellung der armenischen Chronik des Edessener Priesters Matthäus gestaltet. ${ }^{+5}$ Matthäus wurde in der 2 . Hälfte des 11. Jahrhunderts geboren und schrieb wohl in der ersten Hälfte des 12. Jahrhunderts bis vor 1140. Er erzählt ausführlich, interpretiert und vergleicht. Jerusalem und die Grabeskirche befanden sich durchaus innerhalb seines Horizontes. ${ }^{\text {to }}$ So berichtet er für das Jahr 455 der armenischen Zeitrechnung, was dem Jahr $1007 \mathrm{n}$. Chr. und damit der unmittelbaren Nähe unseres Ereignisses entspricht, von einem scharfen Konflikt zwischen Armeniern und Griechen um das Osterdatum: Man habe in Jerusalem das Osterfest zum falschen Datum gefeiert. Deshalb sei in diesem Jahr das Wunder des Osterfeuers in der Grabeskirche ausgeblieben.

Die anwesenden Muslime, die gewöhnlich das Ereignis zusammen mit den Christen begingen, hätten darauthin (offenbar aus Enttäuschung) in der Kirche ein Blutbad unter den Christen angerichtet. Jetzt habe sich der Kaiser Basilius II (958-1025) über die Ursachen informiert. Man habe den Streit um das Osterdatum schließlich in öffentlichen Disputationen zwischen griechischen und armenischen Gelehrten in Konstantinopel ausgefochten. Dazu seien auch jüdische Gelehrte geladen worden, von denen der eine die griechische und der andere die armenische Position unterstützt habe. Letztere sei vom Kaiser approbiert worden. ${ }^{77}$ Hier findet sich damit eine bisher unbeachtete armenische Quelle für die 'Tatsache, dass mindestens bis zur Zerstörung der Grabeskirche Muslime in der Osternacht in der Grabeskirche anwesend waren; von diesen Ausschreitungen ist allerdings andernorts $\mathrm{m}$. W. nicht die Rede. ${ }^{48}$

44 Zu dieser Chronik aktuell Weltecke, Michael Chronik (s. Anm. 8) 192-194; K. Pinggéra, Nestorianische Weltchronistik. Johannes bar Penkāyē und Elias von Nisibis, in: M. Wallfraff ( $\mathrm{Hg}$.), Julius Africanus und die christliche Weltchronistik, Berlin 2006, 263-284.

45 A.E. Dostourian (comm. and introd.), Armenia and the crusades: 10th to 12th centuries - the chronicle of Matthew of Edessa, transl. from the orig. Armenian, Lanham etc. 1993 (Armenian beritage series).

46 Vgl. zu Matthäus die Einleitung zu Dostourian, Chronicle of Matthew (s. Anm. 45); vgl. R. W. 'Thomson, 'The Crusaders through Armenian Eyes, in: A. E. Laiou - R. P. Mottahedeh (Hg.), T'he Crusades from the Perspective of Byzantium and the Muslim World, Washington 2001, 71-82.

47 Dostourian, Chronicle of Matthew (s. Anm. 45) 41f.

48 Dostourian, Chronicle of Matthew (s. Anm. 45) 42.
Hingegen wird der Streit um das Osterdatum im Jahr 1007 auch in der griechisch-orthodoxen Chronik des schon erwähnten Melkiten Yalyya von Antiochia erwähnt, und zwar mit konkreteren Angaben zum Streit selbst. "4 Auch hier sind Juden beteiligt. Yahya führt den Streit sogar direkt auf eine Auseinandersetzung der jüdischen Gelehrten um die Pessachfestberechnung in diesem Jahr zurück. Da das Osterfest in den östlichen Kirchen stets am Sonntag nach Pessach stattzufinden hatte, habe deren Uneinigkeit auch einen Datumsstreit für die Christen nach sich gezogen. Yahya behauptet, dass alle Konfessionen -- er nennt die Melkiten, die Kirche des Ostens und die Miaphysiten - sich sowohl in Syrien als auch in Ägpyten einigen konnten und ihre Entscheidung auch vom melkitischen Patriarchen von Alexandria approbiert wurde. Man hatte sich umgekehrt einer der beiden jüdischen Parteien angeschlossen..$^{50}$ Nur die Jerusalemer Kirche - zu dieser Zeit vakant - und eine Gruppe Miaphysiten in Oberägypten habe sich diesem Entscheid zunächst nicht beugen wollen. Die Jerusalemer aßen damit in der Karwoche noch Fleisch, unterwarfen sich aber schließlich den Entscheidungen und schlossen sich ebenfalls dem Fasten an. ${ }^{51}$ Yahya kündigt an, über die möglichen Zweifelsfälle in der Osterfestberechnung eigens eine Abhandlung verfassen zu wollen, die allerdings an dieser Stelle die Erzählung stören würde. ${ }^{52}$ Matthäus und Yabya datierten den Streit übereinstimmend auf zwei Jahre vor der Zerstörung der Grabeskirche in Jerusalem. Ein solcher innerchristlicher Streit konnte, wie erwähnt, die religiöse Instabilität erhöhen, die vor allem für die Ohimmis bedrohlich werden konnte. Er zeigt zugleich wiederum interreligiöse Verflechtungen in dieser Zeit an, die direkte Auswirkungen auf das religiöse Leben und auf religiöse Entscheidungen hatten.

Matthäus verfügte also durchaus über Informationen zur Grabeskirche. Umso überraschender ist es, dass Matthäus von Edessa die Zerstörung der Grabeskirche selbst oder auch nur den Kalif al-Häkim mit keinem einzigen Wort erwähnt. Dabei hätte ihm diese Zerstörung als Folge des Osterstreits in seiner antigriechischen Polemik (von der er den Kaiser ausnimmt) gut zupass kommen können. Anders als Yahya schweigt er auch zu Christenverfolgungen in Ägypten oder Syrien.

49 Kratchkovsky-Vasiliev, Histoire de Yahya (s. Anm. 15) 273-276.

50 Kratchkovsky-Vasiliev, Histoire de Yahya (s. Anm. 15) 275.

51 Kratchkovsky-Vasiliev, Histoire de Yahya (s. Anm. 15) 276.

52 Kratchkovsky-Vasiliev, Histoire de Yahya (s. Anm. 15) 277. Dieser Osterstreit scheint bisher nicht untersucht worden zu sein. 


\section{Michael der Große}

Allerdings ist Matthäus nicht der einzige Miaphysit der syrisch-mesopotamischen Region, der keine Nachricht zu diesen Ereignissen aufzeichnet. Auch der erst in der 2. Hälfte des 12. Jahrhunderts schreibende syrisch-orthodoxe Patriarch Michael (1126-1199) erwähnt die Zerstörung der Grabeskirche nicht. ${ }^{53}$ Für die syrisch-orthodoxe Kirche fiel das Ereignis von 1009 allerdings in eine Zeit magerer Geschichtsschreibung, die vom 9. bis 11. Jahrhundert andauerte. ${ }^{54}$ Erst Ende des 11. Jahrhundert wurde die chronistische Tradition wieder aufgegriffen. Für diese karge Zeit begnügte sich Michael mit summierenden Angaben oder Exzerpten etwa aus ciner Klostergeschichte; längere Zeiträume konnte er mit keiner Mitteilung füllen, obwohl er eigene Quellenheuristik betrieb und Quellen anderer Konfessionen und Religionen auswertete. ${ }^{55}$ Möglicherweise lagen ihm also keine Nachrichten vor, auch wenn sich unten zeigen wird, dass dies überraschen muss. Doch auch die armenische Bearbeitung dieser Chronik erwähnt das Ereignis nicht. ${ }^{56}$ Sie wurde anfangs des 13. Jahrhunderts in zwei verschiedenen Versionen geschrieben und flocht nochmals viele neue Quellen in das Werk ein. ${ }^{57} \mathrm{Da}$ sie eigenständig mit dem Material verfuhr, muss sie als die dritte miaphysitische Chronik genannt werden, die die Zerstörung der Grabeskirche mit keinem Wort erwähnt.

Die Zerstörung der Grabeskirche fällt in die Amtszeit des syrisch-orthodoxen Patriarchen Johannes bar 'Abdūn (1004-1033). Der Bericht über diese Periode wird bei Patriarch Michael völlig von den Konflikten zwischen der Reichskirche und der syrisch-orthoxen Kirche dominiert, besonders von dem langen Exil des Patriarchen und seinem Tod in Konstantinopel unter Kaiser Romanos III. (1028-1034). ${ }^{58}$ Es sei bemerkt, dass diese für die syrisch-orthodoxe Kirche sehr bedrückenden Ereignisse auch den Kopten Bischof Michael von Tinnis intensiv beschäftigten. Wie er mitteilt, hatte er selbst den syrisch-orthodoxen Patriarchen als Gesandter getroffen. ${ }^{59}$ Bischof Michael sieht hier Parallelen im Geschehen.

53 J.-B. Chabot ( $\mathrm{Hg}$.$) , Chronique de Michel le Syrien. Patriarche Jacobite d'Antiocke$ (1166-1199), I-IV, Paris 1899-1924, Ndr. Brüssel 1963.

54 Weltecke, Michael Chronik (s. Anm. 8) 45f., mit Literatur und Quellen.

55 Weltecke, Michacl Chronik (s. Anm. 8) 133-152.

56 V. Langlois, Chronique de Michel le Grand, patriarche des syriens jacobites. Traduite pour la premier fois sur la version du prêtre Ischôk, Venedig 1868.

57 A. Schmidt, Dic zweifache armenische Rezension der syrischen Chronik Michaels des Großen, in: Mus 109 (1996) 299-319.

58 Ausführlich behandelt von T. Benner, Die syrisch-jakobitische Kirche unter byzantinischer Herrschaft im 10. und 11. Jahrhundert, Marburg 1989, der dazu intensiv Kratchkovsky-Vasiliev, Histoire de Yahya (s. Anm. 15) genutzt hat.

59 Atiya - Abd al-Masị - Burmester, History (s. Anm. 13) 222 f.
Aus seiner Sicht erleiden der syrisch-orthodoxe und der koptische Patriarch dieselben Versuchungen und Mühen, der eine unter dem griechischen König und dessen Patriarch, der andere unter dem König der Muslime und den Bewohnern scines Reiches. ${ }^{60}$ Beide Patriarchen seien heilig und hätten viele Wunder gewirkt, die er am Schluss seines Berichtes eigens aufzählt." ${ }^{61}$ Damit muss man davon ausgehen, dass nach den Ereignissen in Ägypten zwischen den Syrisch-Orthodoxen und den Kopten durchaus ein Austausch stattgefunden hat. Es ist atch wegen des Pilgerverkehrs unwahrscheinlich, dass die Syrisch-Orthodoxen nichts von Ägypten und dem Schicksal der Grabeskirche - wie zu Patriarch Michacls Zeit auch von ihrem Wiederaufbau - gehört hatten.

\section{Anonyme Chronik zum Jahr 1234}

Wie auch immer das Schweigen Michaels und das der Armenier zu erklären wäreein anderer syrisch-orthodoxer Chronist notiert das Ereignis. Ende des 12. und Anfang des 13. Jahrhunderts schrieb der anonyme syrisch-orthodoxe Chronist bis zum Jahr 1234.62 Anders als in den anderen Berichten findet sich bei thm eine genaue Aufzählung der Elemente des spätantiken Komplexes der Grabeskirche. Der Anonymus teilt mit, der Kalif habe die Demolierung „der Kuppel der Anastasis, Golgatas und der Kirche Konstantins" angeordnet. Wenn also Batuntersuchungen die Säulen der Rotunde noch als antik erweisen, haben sie im Anonymus eine stützende Schriftquelle. Im Unterschied dazu ging seiner Ansicht nach die Basilika nun unter. ${ }^{63}$

In seinem kirchengeschichtlichen Teil wiederholt er diese Aufzählung und lässt folgen „und auch das Heilige Grab unseres Herrn Christus“. Zweierlei fügt er hier hinzu. Zunächst datiert er das Ereignis sehr ausführlich: Es geschieht in den Jahren des Patriarchen von Antiochia, Johannes bar 'Abdün und den Tágen des Patriarchen Zacharias von Ägypten und den Tagen des Bischofs 'Thomas von Jersualem. Damit exhält dieses Ereignis cine besondere Markierung. „... sie (die Muslime) warfen alle Steine ins Meer, damit die Christen nicht beten können,

60 Atiya - Abd al-Masīh-Burmester, History (s. Anm. 113) 223.

61 Atiya - Abd al-Masih - Burmester, History (s. Anm. 13) 223-228.

62 Zum Forschungsstand zu dieser Chronik D. Weltecke, Les trois grandes chroniques syro-orthodoxes des XIIe et XIMc siècles, in: M. Debié (Hg.), L'historiographie syriaque, Paris 2009 (Études syriaques 6), 107-135.

63 J.-B. Chabot $(\mathrm{Hg}$.), Anonymi Auctoris chronicon ad annum Christi 1234 pertinens (CSCO 82, Scriptores Syri 37), S. 42 (syr.); A. Abouna (Üs.), Anonymi Auctoris chronicon ad a. C. 1234 pertinens, II, introd., notes et index de J.-M. Fiey (CSCO, 354, Scriptores Syri 154), S. 30 (frz.). 
weil die Heere der Franken davon erfuhren und in Bewegung gesetzt wurden auszuziehen zum Grab des Herrn, was sich in dieser selben Zeit ereignete. "64 Offenbar ist der Bericht mit späteren Exeignissen, den Kreuzzïgen, kontaminiert, die von den syrischen und armenischen Christen in der Hauptsache als Rachefeldzüge der Franken für Übergriffe auf ihre Pilger gedeutet wurden. ${ }^{65} \mathrm{Der}$ Anonymus bringt die Zerstörung der Grabeskirche unmittelbar mit dem Beginn der Kreuzzüge in Verbindung, wenn er sie auch nicht als Auslöser betrachtet.

\section{Bar`Ebrōyō}

Wenige Jahrzehnte nach dem anonymen Chronisten schreibt der letzte Autor, der hier erörtert werden soll. Sein Bericht wurde bereits von Canard angesprochen. ${ }^{65}$ Es ist der Primas des östlichen Teils der syrisch-orthodoxen Kirche, der Maphrian Bar 'Ebröyö (1226-1286), der eine große syrische Weltchronik mit einem weltlichen und zwei kirchlichen Abschnitten verfasste sowie eine kurze arabische Version davon, der er ein etwas anderes Profil gegeben hat. ${ }^{67}$ Er notiert im weltichen 'Teil seiner syrischen Chronik, dass der Kalif al-Hakim die Kirche der Anastasis von den Fundamenten her zerstört habe. ${ }^{68}$

Sein Bericht macht die interreligiöse Verflechtung in der ägyptischen Gesellschaft und die besondere Rolle der christlichen Beamten wieder besonders deutlich. Gleich im Zusammenhang mit al-Hākims Regierungsantritt bemerkt Bar 'Ebrōyō, dass damals Christen noch hatten Wezire werden können. Heute müssten sie hingegen zum Islam übertreten, wenn sie dies anstrebten. ${ }^{69}$ Über die Schwester des Kalifen konnten indessen immer noch selbst niedrigere koptische Beamte einen Emir stürzen. ${ }^{70}$ Allerdings löste ein hoher christlicher Beamter zu

64 Abouna, Anonymi Auctoris 1234 II CE (s. Anm. 63) S. 284f. (214).

65 Immer noch Standard: A. Lüders, Die Kreuzzüge im Urteil syrischer und armenischer Quellen, Berlin 1964.

66 Canard, Destruction (s. Anm. 2) 25.

67 Zum Forschungsstand und zur aktuellen Literatur Hidemi Takahashi, Barhebraeus. A Bio-Bibliography, Ncw Jersey 2005; ergänzend Weltecke, Les trois chroniques (s. Anm. 62).

68 Bedjan, Barhebraei Chronicon / Budge, Chronography Bar Hebraeus (s. Anm. 30) S. 204 (184).

69 Bedjan, Barhebraei Chronicon / Budge, Chronography Bar Hebraeus (s. Anm. 30) S. $200(180)$.

70 Bar 'Ebrōyō, Chronik (s. Anm. 30) S. 202f. (182). Dies ist in mehreren arabischen Quellen erwähnt und wohl von hier zu Bar'Ebrōyō gekommen, vgl. L. Yaakov, T'he Fāțmid Princess Sitt al-Mulk, in: Journal of Semitic Studies 32, 2 (1987) 319-328, hiex: 322.
Beginn der Herrschaft al-Häkims bereits Verfolgungen aus, weil er den Zorn des ägyptischen Adels erregt habe. Vicle Notare (nümüqē) seien wegen ihm geschlagen worden. ${ }^{71}$ Diese Ereignisse werden zu Christenverfolgungen im Vorderen Orient parallel gesetzt, wenn auch nicht in Verbindung gebracht. So sei es im Jahr 1001 n. Chr. auch in Bagdad zu Ausschreitungen gegen Christen gekommen. Insbesondere eine syrisch-orthodoxe Kirche sei bei dieser Gelegenheit zerstört worden und habe viele Menschen auf dem daneben gelegenen Mahlplatz unter sich begraben. ${ }^{72}$

Bar 'Ebröyö inseriert cine Version des Befehls, mit dem Christen und Juden gezwungen werden sollten, sich öffentlich mit hölzernen Kreuzen und Kugeln zu kennzeichnen. Auch die Ausreise nach Byzanz sei den Christen mhegelegt worden, die viele auf sich genommen hätten. Dagegen hätten nur wenige dem Glauben abgeschworen. ${ }^{73}$ Mit der Beschreibung der Kennzeichen und der Begründung, die der Kalif für sie gibt, stimmt er mit den Ausführungen des Michael von Tinnis überein. ${ }^{74}$ Sie werden hier allerdings sehr viel ausdrücklicher als entehrende Schandmale markiert. Aus Bar 'Ebrōyōs Sicht war es dem Kalifen nicht nur um eine bessere Unterscheidung zwischen Christen und Muslimen zu tun.

Bar 'Ebrōyō ist außer Michael von 'Tinnis der Einzige, der in seiner Darstellung eine Interpretation und einen Auslöser für diese Ereignisse vorstellt. Bei ihm ist es allerdings nicht die Schuld der Kopten, sondern die eines Mannes, der die Christen hasste. ${ }^{75}$ Dies fügt sich zum einen in die für Christen allgemein als gefährlich beschriebene Situation ein und zum anderen zu der beschriebenen Neigung des Kalifen, allen möglichen Anschuldigungen sein Ohr zu leihen und entsprechend umgehende Maßnahmen zu ergreifen.

Dieser Mann habe dem Kalifen erläutert, wie die Christen das Heilige Feuer der Osternacht fälschten: Wenn der Emir von Jerusalem die Tür des Grabes geschlossen habe, ließen die Christen über einen zuvor eingeölten Draht von oben das Feter in die Lampe herabkommen, die über dem Grab hing. Dann riefen sie „Kyrie Eleison“ und weinten und gäben vor, ein Licht zu sehen, das vom Himmel auf das Grab herabkomme. Dies ist der einzige Beleg für die Fäl-

71 Bedjan, Barhebraei Chronicon / Budge, Chronography Bar Hebraeus (s. Anm. 30) S. $203(182)$.

72 Bedjan, Barhebraei Chronicon / Budge, Chronography Bar Hebraeus (s. Anm. 30) S. $203(183)$.

73 Bedjan, Barhebraei Chronicon / Budge, Chronography Bar Hebracus (s. Anm. 30) S. 205f (184).

74 Bedjan, Barhebraei Chronicon / Budge, Chronography Bar Hebraeus (s. Anm. 30) S. 204 (184).

75 Bedjan, Barkebraei Chronicon / Budge, Chronography Bar Hebraeus (s. Anm. 30) S. $205(185)$ 
schungsgeschichte aus christlicher Feder. Doch scheint es wahrscheinlich, dass Bar 'Ebröyō ihn einer muslimischen Quelle entnommen hat." $\mathrm{Er}$ fährt allerdings fort, dass der Kalif am Ende seines Lebens bereut habe, die Juden und Christen bedrückt zu haben. Er habe den Zwangsmuslimen erlaubt, zum Christentum zurückzukchren. Alle Christen durften ihre Kirchen wieder errichten. Noch dazu hätten sie nun schönere Kirchen als vorher gebaut. Auch seien die Flüchtlinge aus Byzanz zurückgekehrt. ${ }^{77}$

Wie Michael von Tinnis berichtet auch er von den einschränkenden und diskriminierenden Maßnahmen gegen die Frauen, sogar in noch größerem Detail, allerdings erst im Anschluss an den Bericht zur Grabeskirche. Er erzählt, bald seien die wirtschaftlichen Probleme sichtbar geworden, die die Ausgangssperre für die Frauen mit sich brachte. Deshalb sei für allein lebende Witwen auf dem Land nach Beschwerden ein männlicher $\mathrm{Hol}$ - und Bringdienst eingerichtet worden. Dieser habe die Handwerkserzeugnisse der Frauen an deren Haustuir abgeholt und sie stellvertretend auf dem Markt verkauft. ${ }^{78}$

Am Schluss seines Berichtes erzählt er vom Verschwinden des Kalifen als Mordkomplott seiner Schwester, Sitt al-Mulk. Dieses Komplott wurde von einigen muslimischen Autoren vermutet, aber von niemandem so ausgestaltet erzähl wie in der sunnitischen Polemik im Geschichtswetk des Hilăl aș-Ṣăbi (969-1056). Dieses Werk ist allerdings nur in späteren Darstellungen fragmentarisch überliefert." Doch ist es diese Version, die offenbar auch Bar 'Ebrōyo kennt. Die Schwester sei mittels einer lebensgroßen Puppe öffentlich der Unzucht angeklagt worden. Sie habe gefürchtet, ihr Bruder könne Hebammen schicken, um ihre Jungfräulichkeit zu kontrollieren. Darauthin habe sie die Verschwörung zur Ermordung des Kalifen ins Werk gesetzt. ${ }^{80}$

76 Vgl. die Zusammenstellung von Canard, Destruction (s. Anm. 2) 20-23. Der wahrscheinliche Ursprung der Information scheint mir Hiläl aş-Săbi zu scin, doch über welche Vermittlung, war hier nicht zu ermitteln, s. auch unten.

77 Bedjan, Barhebraei Chronicon / Budge, Chronography Bar Hebraeus (s. Anm. 30) S. $205(185)$.

78 Bedjan, Barhebraci Chronicon / Budge, Chronography Bar Hebraeus (s. Anm. 30) S. $206 f .(185 f$.$) .$

79 H. Halm, Dic Kalifen von Kairo. Die Fatimiden in Ägypten 973-1074, München 2003 300, bezeichnet dessen Darstellung als "dubios". Eine ausführliche Diskussion zu der Version Flläls und den überliefertek Fragmenten bietet Halm, Treuhänder (s. Anm. 17) 66-70, und Yaakov, Fătimid Princess (s. Anm. 70) $324 \mathrm{f}$.

80 Bedjan, Barhebraei Chronicon / Budge, Chronography Bar Hebracus (s. Anm. 30) S. 207-210 (187-189). Von solchen Puppen, die die Schwester und den Kalifen schmähen, ist öfter die Rede; auch Michael von 'Tinnis erwähnt sie, allerdings fü: cine frühere Zeit, Atiya - Abd al-Masih - Burmester, History (s. Anm. 13) 92.
Nur ist es fraglich, ob Bar 'Ebrōyō diese Geschichte direkt oder in muslimischer oder christlicher Vermittlung kennenlernte. Denn der Schluss seines Berichtes ist widersprüchlich. Zunächst stellt er fest, dass wegen der Eigenart des nächtlichen Mordes, bei dem der Leichnam nie gefunden wurde, viele geglaubt hatten, der Kalif habe sich in die Einöde zurückgezogen, um Mönch zu werden. Ähnliches behaupteten auch koptische Notare Bar 'Ebröyö gegenüber, als er in Damaskus weilte. Sie sagten, dem Kalifen sei während der Verfolgung Christus offenbart worden; er sei Christ geworden und heimlich in die Wüste aufgebrochen. ${ }^{81}$ Wie Bar 'Ebröyö in seiner Kirchengeschichte berichtet, hielt er sich 1258 im Auftrag seines Patriarchen am ayyubidischen Hof in Damaskus auf und hatte dort intensiven Kontakt zu hohen koptischen Beamten. ${ }^{82}$ Anscheinend sind bei dieser Gelegenheit auch historische Gegebenheiten zur Sprache gekommen, eine Abendunterhaltung, die er auch sonst für christliche Intellektuelle, weltliche wie kirchliche, beschreibt. ${ }^{83}$ Auf diese Bemerkung lässt er unvermittelt die Meinung derjenigen folgen, die den Kalifen mit den sich selbst vergöttlichenden und daher frevelnden Pharaonen gleichsetzten. ${ }^{84}$ Dieses letztere entspricht wieder eher der stumitischen Polemilk.

Anders als bei Michael von 'T"innis geht bei Bar. 'Ebröyö die Katastrophe von eifersüchtigen Muslimen aus, die auch sonst in seiner Chronik immer wieder eine für die Christen gefährliche Rolle spielen. In der Mitte des 13. Jahrhunderts haben sich die Verhältnisse zwischen Christen und Muslimen und die Machtpositionen, die sie einnehmen können, zuungunsten der Christen verschoben. Trotzdem ist auch seine Darstellung des Kalifen ambivalent. Doch scheint er selbst dabei unbeteiligt. Er gibt sich neutral und scheint nur zu zitieren. Sein Bericht geht nicht mehr direkt auf zeitgenössische Quellen zurück. Er ist ein bisher noch unentwirrtes Geflecht aus unterschiedlichen Quellen und Erzählschichten, der Polemik und christliche Erinnerungsarbeit gleichermaßen enthält. Trotz inhaltlicher Übereinstimmungen hat sein Bericht keine direkten Beziehungen zur koptischen Patriarchengeschichte und belegt somit eine weitere Tradition.

81 Bedjan, Barhebraci Chronicon / Budge, Chronography Bar Kebraeus (s. Anm. 30) S. $210(189)$.

82 Abeloos - Lamy, Gregorii Barhebrei (s. Anm. 39), II, S. 711-722 (712-723), III, S. 427 (428).

83 Abeloos - Lamy, Gregorii Barhebrci (s. Anm. 39), I, S. 637; 697; 745; 771 (638; 698; 746; 772); Bedjan, Barhebraei Chronicon / Budge, Chronography Bar Hebraeus (s. Anm. 30) S. 457 (392)

84 Bedjan, Barhebraci Chronicon / Budge, Chronography Bar Hebracus (s. Anm. 30) S. $210(189)$. 


\section{Schluss}

Unbefriedigend bleibt für den Augenblick der Grad der heuristischen und quellenkritischen Analyse zu den orientchristlichen Quellen, die voranzutreiben sich lohnen würde. Indessen lassen sich vorläufig folgende Gesichtspunkte ableiten: Im Augenblick scheint sich eine wissenschaftliche Neubewertung des Kalifen anzubahnen. Doch sei hier betont, dass zumindest die Berichte der orientalischen Christen voneinander unabhängig, eindeutig und zuverlässig genug sind. Die entehrenden, diskriminierenden und einschränkenden $\mathrm{Maßnahmen} \mathrm{gegen} \mathrm{Chris-}$ ten und Juden und die Zerstörung (wichtiger Teile) der Grabeskirche haben stattgefunden. Weil die Maßnahmen am Ende der Herrschaft wieder aufgehoben wurden und eine Restauration eingeleitet worden ist, ist der Kalif gleichwohl nicht als Monster, sondern als widersprüchliche, auch ambivalente Figur in die christliche (wie die jüdische) Erinnerung eingegangen. Sein radikal asketisches Verhalten wird dabei zum einen als besondere muslimische Frömmigkeit interpretiert, zum anderen jedoch von den orientalischen Christen nicht als wahnhaft, sondern als christianisierendes Verhalten verstanden. Sie überlieferten nicht zuletzt enge Bezichungen zu christlichen Mönchen. Und auch von orientchristlicher Seite konnte man bezweifeln, dass der Kalif tatsächlich ermordet worden war. Dann hätte er als christlicher Asket in der Wüste sein Leben beschlossen.

Was die orientalischen Stellungnahmen betrifft, ist daneben ein weiterer, auffälliger Unterschied zur lateinischen Welt zu konstatieren. Es fehlt, auf dieses Ereignis bezogen, jedes antijüdische Ressentiment. Die orientalischen Christen berichten, dass Juden wie Christen gleichermaßen betroffen waren. Es gibt bei ihnen keine verschwörerischen Verbindungen zwischen Juden und dem Kalifen al-Häkim. Als Auslöser wurde einmal ein koptischer Mönch genannt, einmal ein muslimischer Christenfeind. Weder wurde eine allgemeine Verschwörungstheorie konstruiert, noch eine reine Christenverfolgung als solche konstruiert. Darüber hinaus werden zwar in diesen Werken die Zeichen des Himmels beachtet, doch war keine verbreitete eschatologische Stimmung zu konstatieren. Vor allem fehlten Hinweise dafür, dass die orientalischen Christen annahmen, al-Häkim handele aus einer eschatologischen Naherwartung heraus. Wo er verglichen wird, ist er Pharao, nicht der Antichrist. Man kann diesen Autoren auch beileibe keine allgemeine antimuslimische T'endenz unterstellen. Darüber hinaus hatte etwa der Zeitzeuge Michael von Tinnis das Ereignis mit dem Exil des syrisch-orthodoxen Patriarchen in Byzanz parallelisiert.

Aus diesen Quellen lassen sich insgesamt wohl keine zusätzlichen Informationen über die Auslöser der Zerstörung der Grabeskirche gewinnen. Allerdings betten die orientalischen Christen das Ereignis in den Zusammenhang der Religionspolitik des Kalifen insgesamt ein, zu der auch seine innermuslimischen
Maßnahmen gehören. Außerdem verweisen sie auf eine eher regionale als interkonfessionelle religiöse Unruhe, die sich im Streit um das Osterfest im Jahr 1007 entlud. Im Rahmen der Rückeroberungen der Byzantiner im 10. Jahrhundert im kilikischen, syrischen und mesopotamischen Raum wurden die Christen mit der Macht des Kaisers und der orthodoxen Kirche konfrontiert; neue und alte Konflikte flackerten mit neuer Schärfe auf.

Die Zerstörung der Grabeskirche scheint daneben für die orientalischen Christen nur eine Episode zu sein, die sich ähnlichen Episoden, nämlich zeitweiligen Ausschreitungen gegen Christen, die zuweilen von außen, zu anderen Zeiten auch von den Christen selbst ausgelöst sein konnten, hinzugesellt. Sie markiert keine Zeitwende. Auch andere Kirchen wurden in dieser Zeit zerstört. Dies war eher eine wiederkehrende Erfahrung. Allerdings verknüpfte der anonyme Chronist bis 1234 bemerkenswerterweise im Rückblick die Zerstörung der Grabeskirche mit dem Aufbruch der Lateiner aus dem Westen. Doch haben andere Begebenheiten sehr viel tiefere Einschnitte in der historischen Erinnerung orientalischer Christen hintedlassen als die Zerstörung der Grabeskirche. Wieder sei Edessa erwähnt und seine zweifache Eroberung im Jahr 1144 und 1146. Dic Reaktionen zur Zerstörung der Grabeskirche ähneln in nichts dem Aufschrei, der aus armenischen und syrischen Quellen für diese Katastrophe belegt ist. ${ }^{85}$ Wenn auch die symbolische und spirituelle Bedeutung Jerusalems zu dieser Zeit für die orientalischen Christen außer Frage steht; die physische Grabeskirche lag ihnen offenbar nicht in derselben Weise am Herzen.

Unterstrichen sei am Schluss ein Element, das die Berichte durchzicht und das in der Tat auf die besondere historische Situation um 1000 verweist. Zwar bestätigen die orientchristlichen Quellen insgesamt nicht Marius Canards Vermutung, dass die islamische Teilnahme am Osterfest in der Grabeskirche oder die Betrugsbeschuldigungen die Zerstörung ausgelöst haben. Dazu ist der einzige Bericht, der dies ausdrücklich behauptet, nicht unabhängig genug. Aber es sind die vielfältigen Verflechtungen zwischen den Konfessionen und den Religionen, sei es in der Grabeskirche, sei es in Ägypten oder andernorts, die gerade in den frühen Quellen ohne erzählerische Absicht sehr deutlich werden. Aus der Perspektive des 13. Jahrhunderts begegnet man hier einer anderen Welt, wie es auch

85 T. M. van L.int, Lament on Edessa by Nersēs Šnorhali, in: K. Ciggaar - H. Teule (H.J.), East and West in the Crusader States: Context - contacts - Confrontations, II. Actal of the congress held at Hernen Castle in May 1997, Leuven 1999, 49-105; D. Weltecke, Überlegungen zu den Krisen der syrisch-orthodoxen Kirche im 12. Jahrhundert, in: M. 'Tamcke (Hg.), Syriaca. Zur Geschichte, Theologie, Liturgie und Gegewartslage der syrischen Kirchen, 2. Deutsches Syrologen-Symposium (Juli 2000, Wittenberg), Münster - Hamburg - London 2002 (Studien zur Orientalischen Kirche 17), 125-145. 
der Maphrian Bar 'Ebröyö bemerkt. Doch die Zeitgenossen des frühen 11. Jahrhunderts scheinen sich darüber nicht zu wundern. Insofern ist der Kalif mit seinem Bestreben, die Religionen und die Geschlechter in eine klar erkennbare und hicrarchische Ordnung $z u$ bringen, ein Vorbote der Zukunft, die ihn an Radikalität weit übertreffen wird. 